\title{
ON 3-MANIFOLDS THAT HAVE FINITE FUNDAMENTAL GROUP AND CONTAIN KLEIN BOTTLES
}

BY

\author{
J. H. RUBINSTEIN ${ }^{1}$
}

\begin{abstract}
The closed irreducible 3-manifolds with finite fundamental group and containing an embedded Klein bottle can be identified with certain Seifert fibre spaces. We calculate the isotopy classes of homeomorphisms of such 3-manifolds. Also we prove that a free involution acting on a manifold of this type, gives as quotient either a lens space or a manifold in this class. As a corollary it follows that a free action of $Z_{8}$ or a generalized quaternionic group on $S^{3}$ is equivalent to an orthogonal action.
\end{abstract}

0. Introduction. We are in the PL category. The object of study is the class of closed, irreducible orientable 3-manifolds which contain embedded Klein bottles and have finite fundamental group. These 3-manifolds are easily shown to be exactly the Seifert fibre spaces [7] with at most 3 exceptional fibres of multiplicity $2,2, p(p \geqslant 1)$ and the 2-sphere as orbit surface, excluding $S^{2} \times S^{1}$.

We prove that any homeomorphism homotopic to the identity is isotopic to the identity for such a 3-manifold $M$ (this was done for a particular case where $p=2$ in [4]). Also the factor group of the group of orientation-preserving homeomorphisms of $\boldsymbol{M}$ by the normal subgroup of homeomorphisms isotopic to the identity, which is denoted $\mathcal{H}(M)$, is shown to be one of the groups $Z_{2}, Z_{2}+Z_{2}, S_{3}$ and $S_{3}+Z_{2}$. There are no orientation-reversing homeomorphisms of $M$.

Finally we establish that any free involution on $M$ gives as quotient either a lens space or a 3-manifold in the above class. Let $Q(8 \mathrm{~m})$ be the group $\left\{x, y \mid x^{2}=(x y)^{2}=y^{2 m}\right\}$. As a corollary it follows that a free action of $Q\left(2^{k}\right)$ on $S^{3}, k>3$, is equivalent to an orthogonal action. Also simpler proofs of the analogous result in [5] and [6] for $Z_{4}$ and $Z_{8}$ are given.

Note that the 3-manifolds in the above class are not sufficiently large. Therefore it is interesting to see that some of the results of Waldhausen [9] can be achieved in this case. In another paper [11] we will build on the work here to obtain that free actions of some finite groups of order $2^{m} 3^{n}$ on $S^{3}$ are equivalent to orthogonal actions.

Received by the editors November 24, 1976 and, in revised form, March 20, 1978.

AMS (MOS) subject classifications (1970). Primary 57A10, 57E05, 57E25; Secondary 55A10. Key words and phrases. Seifert fibre space, isotopy class of homeomorphisms, free group action.

1 The author held a Rothman's Fellowship during this research. 
Note added during revision. Similar results to $\$ 1$ have been obtained by $\mathrm{P}$. Kim, to $\$ 2$ by K. Asano, S. Cappell and J. Shaneson and to $\$ 3$ by B. Evans and J. Maxwell. I would like to thank the referee for his suggestions and improvements to the paper.

\section{Seifert spaces.}

Definition. A closed surface $J$ embedded in a 3-manifold $M$ is incompressible if (1) $J$ is a 2-sphere and $J$ does not bound a 3-cell or (2) $J$ is not a 2-sphere and there is no disk $D$ embedded in $M$ with $D \cap J=\partial D$ a noncontractible curve in $J$.

LEMMA 1. Let $K$ be a Klein bottle. Then there are exactly five isotopy classes of simple closed curves in $K$. If $\pi_{1}(K)=\left\{a, b \mid b^{-1} a b=a^{-1}\right\}$ then these are represented by $\{1\}, a, b, a b, b^{2}$.

Proof. See [4].

Let $M$ be a closed, irreducible orientable 3-manifold with finite fundamental group and $K$ be an embedded Klein bottle in $M$. Since $M$ is orientable, $K$ must be one-sided in $M$. We denote a small regular neighbourhood of $K$ by $N$. Finally let $Y=M-$ int $N$ and denote $\partial Y=\partial N$ by $L$.

LEMMA 2. $K$ is incompressible and $Y$ is a solid torus.

Proof. Suppose that $K$ is compressible in $M$ and let $D$ be a disk with $D \cap K=\partial D=C$ noncontractible in $K$. Then $C$ is two-sided in $K$ and therefore either is a nonseparating curve on $K$ or divides $K$ into two Möbius bands (cf. Lemma 1). Let $N(D)$ be a small regular neighbourhood of $D$, which intersects $K$ in an annulus $A$. Let $D_{0}$ and $D_{1}$ be the two disjoint disks in $\partial N(D)$ with $\partial D_{0} \cup \partial D_{1}=\partial A$. If we replace $K$ by $(K-$ int $A) \cup D_{0} \cup D_{1}$ then the result is either a nonseparating 2-sphere (since $K$ is one-sided) or two disjoint one-sided projective planes in $M$. Both of these possibilities contradict $\pi_{1}(M)$ is finite. So $K$ must be incompressible.

Since $\pi_{1}(M)$ is finite, by Lemma 14.12 of [12] it follows that $Y$ is a handlebody as desired (i.e. a solid torus).

Proposition 3. The class of Seifert spaces with $S^{2}$ as orbit surface and at most 3 exceptional fibres of multiplicity $2,2, p(p>1)$ excluding $S^{2} \times S^{1}$, is equivalent to the class of irreducible 3-manifolds which have finite fundamental group and contain an embedded Klein bottle.

Proof. Suppose $M$ is of the latter type. $\pi_{1}(L)$ has generators given by $a$ and $b^{2}$ in $\pi_{1}(K) . N$ can be fibered by circles which have homotopy class $b^{2}$, with two exceptional fibres of multiplicity 2 at the centres of the Möbius bands on $K$ (with classes $b$ and $a b$ ). Since $K$ is incompressible, the boundary of a meridian disk for $Y$ yields an element of $\pi_{1}(L)$ different from $b^{2}$. So the 
fibering extends to $Y$ with another exceptional fibre of multiplicity $p(p>1)$.

Conversely let $M$ be a Seifert fibre space as in the proposition. If $\lambda$ is a nonsingular arc in the orbit surface, joining the images of the exceptional fibres of multiplicity 2 and missing the image of the other exceptional fibre, then the set of points of $M$ which project to $\lambda$ form a Klein bottle. Since $M$ is not homeomorphic to $S^{1} \times S^{2}$ it follows that $\pi_{1}(M)$ is finite and $M$ has $S^{3}$ as its universal cover. Therefore $M$ is irreducible and the result is proved.

Suppose $M$ is a 3-manifold satisfying the conditions in Proposition 3. Let $D$ be a meridian disk for $Y$ and let $C=\partial D$. Assume the homotopy class $\{C\}=a^{m} b^{2 n}$, where $m, n \geqslant 0$ and $(m, n)=1$. Then $\pi_{1}(M)$ has the presentation $\left\{a, b \mid b^{-1} a b=a^{-1}, a^{m} b^{2 n}=1\right\}$. Since $K$ is incompressible, $m \neq 0$ and $n \neq 0$. Conjugating $a^{m} b^{2 n}=1$ by $b$, we see that $a^{2 m}=b^{4 n}=1$. Let $4 n=2^{k} n_{1}$ where $n_{1}$ is odd, and let $b_{1}$ denote $b^{n_{1}}$. Then $\pi_{1}(M)=Z_{n_{1}} \times G$ where the cyclic group has generator $b^{2^{k}}$ and $G=\left\{a, b_{1} \mid b_{1}^{-1} a b_{1}=a^{-1}, a^{m} b_{1}^{2^{k-1}}=1\right\}$.

If $m$ is odd then $G=D\left(2^{k}, m\right)=\left\{a_{1}, b_{1} \mid b_{1}^{-1} a_{1} b_{1}=a_{1}^{-1}, a_{1}^{m}=1, b_{1}^{2^{k}}=1\right\}$, where $a_{1}=a^{2}$. If $m$ is even then since $(m, n)=1$ it follows that $n$ is odd, $k=2$ and $n_{1}=n$. In this case $G=Q(4 m)=\left\{a, b_{1} \mid b_{1}^{2}=\left(a b_{1}\right)^{2}=a^{m}\right\}$.

In the degenerate case $m=1$, clearly $\pi_{1}(M)=Z_{4 n}$. By $[1], M=L(4 n$, $\pm(2 n-1))$ since $M$ contains a Klein bottle.

2. The homeotopy group. Let $M$ be a 3-manifold with the properties in Proposition 3, throughout this section.

THEOREM 4. If $h: M \rightarrow M$ is any homeomorphism with $h_{\sharp}: H_{1}\left(M, Z_{2}\right) \rightarrow$ $H_{1}\left(M, Z_{2}\right)$ equal to the identity, then $h$ is isotopic to a map taking $K$ to $K$.

Proof. Denote $h(K)$ by $K^{\prime}$ and assume that $K^{\prime}$ and $K$ are transverse. Since $h_{\sharp}=\mathrm{id}, h_{*}: \pi_{1}(M) \rightarrow \pi_{1}(M)$ must preserve the normal subgroup $G$ of index 2 obtained from the orientation-preserving elements of $\pi_{1}(K)$. (Note that commutators in $\pi_{1}(K)$ are orientation-preserving loops.) Since the image of $\pi_{1}(Y)$ in $\pi_{1}(M)$ is clearly $G$, it follows that $K^{\prime} \cap Y$ must be orientable.

By the incompressibility of $K^{\prime}$ and $K$, and the irreducibility of $M$, there is an obvious isotopy of $K^{\prime}$ eliminating all the contractible curves of intersection of $K^{\prime}$ and $K$. Consequently it suffices to suppose that $K^{\prime} \cap Y$ contains annuli only and all the curves of $K^{\prime} \cap L$ are noncontractible and parallel on $L$. By the well-known fact that a properly embedded, incompressible annulus in a solid torus is parallel into the boundary, we can then find an isotopy of $K^{\prime}$ achieving $K^{\prime} \cap Y=\varnothing$.

Let $N^{\prime}$ be a small regular neighbourhood of $K^{\prime}$ in $N$ and let $L^{\prime}=\partial N^{\prime}$. If the map $\pi_{1}\left(L^{\prime}\right) \rightarrow \pi_{1}(N)$ has nontrivial kernel then the argument in Lemma 14.12 of [12] implies that $M$ is contained in $N$, which is impossible. So $L^{\prime}$ is incompressible in $N$, and letting $W=N-$ int $N^{\prime}$ we see that $W$ is an $h$ cobordism. Therefore $W$ is homeomorphic to $S^{1} \times S^{1} \times I$ (cf. [8]) and there 
is an isotopy taking $L^{\prime}$ to $L$. Using [9] we can achieve $K^{\prime}=K$ by another isotopy, since $N$ is sufficiently large.

THEOREM 5. If $h: M \rightarrow M$ is a homeomorphism homotopic to the identity then $h$ is isotopic to the identity.

Proof. By Theorem 4 it suffices to assume $h$ takes $K$ to itself. Suppose $h$ fixes the base point on $K$. Then $h_{*}: \pi_{1}(K) \rightarrow \pi_{1}(K)$ maps $a$ to $a^{ \pm 1}$ and $b$ to $b^{ \pm 1}$ or $(a b)^{ \pm 1}$ without loss of generality, by Lemma 1 . There is an isotopy in $K$ inducing conjugation of $\pi_{1}(K)$ by $b$. This takes $a$ to $a^{-1}$ and so we can assume $h_{*}(a)=a$.

As $h$ is homotopic to the identity, $b$ and $h_{*}(b)$ are conjugate in $\pi_{1}(M)$. Therefore for some element $g, b^{-1} g^{-1} h_{*}(b) g$ is in the normal closure of the relation $r=a^{m} b^{2 n}$ in $\pi_{1}(K)$. By a calculation in $\pi_{1}(K)$, one sees that $g^{-1} h_{*}(b) g=h_{*}(b) a^{2 i}$ for some integer $i$. So

$$
b^{-1} h_{*}(b) a^{2 i}=g_{1}^{-1} r^{ \pm 1} g_{1} g_{2}^{-1} r^{ \pm 1} g_{2} \ldots
$$

Suppose $h_{*}(b)=b^{-1}$ or $(a b)^{-1}$. If we put $a=1$ in $(+)$ then it follows that $n=1$. On the other hand if we assume $h_{*}(b)=a b$ and set $a^{2}=1$ in $(+)$ then this gives a contradiction. Finally in the case that $h_{*}(b)=b, h: K \rightarrow K$ is homotopic to the identity. Therefore by [2], after an isotopy we obtain that $h$ is the identity on $K$. Because $h$ must be orientation-preserving it is easy to isotop $h$ to the identity on $N$ and then on all of $M$.

Assume now that $h_{*}(b)=b^{-1}$ or $(a b)^{-1}$ and $n=1$, i.e., $\{\partial D\}=b^{2} a^{m}$ where $D$ is a meridian disk for $Y$. Then the classes $a$ and $\{\partial D\}$ have intersection number \pm 1 in $L$. We isotop $K$ as follows:

First we can move $K$ till $K \cap Y$ is an annulus $A$ in $L$, with the curves of $\partial A$ having homotopy class $a$. The meridian disk $D$ can be assumed to meet $A$ at a single arc. Therefore $A$ is parallel to $L-$ int $A$ in $Y$ and there is an isotopy of $K$ taking $A$ to $L-$ int $A$. Then $K$ can be shifted back to its original position, by the same argument as at the end of Theorem 4.

Depending on the direction of the isotopy, we see that $b$ is transformed to the class $b\left(b^{2} a^{m}\right)^{ \pm 1}$ in $\pi_{1}(K)$. For the appropriate choice, the result is $b^{-1} a^{-m}$. Consequently if the isotopy is applied to $h$ then a homeomorphism is obtained which takes $b$ to $b a^{p}$ for some $p$. By the previous argument, this is isotopic to the identity as required.

Theorem 6. Let $M$ be a 3-manifold as in Proposition 3. Then

$$
\mathcal{H}(M)= \begin{cases}Z_{2}+Z_{2} & \text { if } m \neq 2 \text { and } n \neq 1, \\ Z_{2} & \text { if } m \neq 2 \text { and } n=1, \\ S_{3}+Z_{2} & \text { if } m=2 \text { and } n \neq 1 \\ S_{3} & \text { if } m=2 \text { and } n=1\end{cases}
$$


There are no orientation-reversing homeomorphisms of $M$.

Proof. Let the map $\mathcal{H}(M) \rightarrow$ Aut $H_{1}\left(M, Z_{2}\right)$ given by $h \rightarrow h_{\sharp}$ have kernel $\mathcal{G}$. By Theorem 4, a homeomorphism $h$ with isotopy class in $\mathcal{G}$ can be assumed to map $K$ to itself. By Lemma 1, without loss of generality $h_{*}$ : $\pi_{1}(K) \rightarrow \pi_{1}(K)$ takes $a$ to $a^{ \pm 1}$ and $b$ to $b^{ \pm 1}$ or $(a b)^{ \pm 1}$. Conversely the homeomorphisms of $K$ which transform the pair $(a, b)$ to one of $(a, b)$, $\left(a^{-1}, b^{-1}\right),(a, a b),\left(a^{-1},(a b)^{-1}\right)$ clearly map $\{\partial D\}$ to $\{\partial D\}^{ \pm 1}$ and so extend to homeomorphisms of $M$. Since there is an isotopy of $K$ taking $a$ to $a^{-1}$ these maps give all possible isotopy classes in $\mathcal{G}$.

Suppose first that $m$ is odd. Then $H_{1}\left(M, Z_{2}\right)=Z_{2}$ and so $\mathcal{G}=\mathcal{H}(M)$. The argument in Theorem 5 shows that no pair of the elements $b^{ \pm 1},(a b)^{ \pm 1}$ are conjugate in $\pi_{1}(M)$ for $n \neq 1$, and so $\mathcal{H}(M)=Z_{2}+Z_{2}$. On the other hand if $n=1$ then a homeomorphism $h$ with $h(K)=K$ and $h_{*}(b)=(a b)^{-1}$ is isotopic to the identity (by the method in Theorem 5). Therefore $\mathcal{H C}(M)=Z_{2}$ in this case.

Assume now that $m$ is even. Then $H_{1}\left(M, Z_{2}\right)=Z_{2}+Z_{2}$ and a homeomorphism $h$ taking $K$ to $K$ with $h_{*}(b)=(a b)^{ \pm 1}$ induces a nontrivial involution in Aut $H_{1}\left(M, Z_{2}\right)$. Therefore the same process as in the previous paragraph shows that $\mathcal{G}=Z_{2}$ if $n \neq 1$ and $\mathcal{G}=\{1\}$ if $n=1$.

Let $\mathcal{G}_{0}$ be the quotient of $\mathcal{H}(M)$ by $\mathcal{G} . \mathcal{G}_{0}$ is isomorphic to the image of $\mathcal{H}(M)$ in Aut $H_{1}\left(M, Z_{2}\right)$ and we already know the latter group contains an element of order 2. So $\mathcal{G}_{0}=Z_{2}$ or $S_{3}$ are the only possibilities. If the latter holds then there is a homeomorphism $h: M \rightarrow M$ with $h_{\sharp} \in$ Aut $H_{1}\left(M, Z_{2}\right)$ of order 3. Assume $h_{*}: \pi_{1}(M) \rightarrow \pi_{1}(M)$ takes $a$ to $a^{i} b^{j}$. Then $a^{i} b^{j}$ must have order $2 m$. Consequently $b^{2 m j}$ is a power of $a$ and so $n$ divides $j$ (since $(m, n)=1$ and $m$ is even). If $j$ is odd then $a^{i} b^{j}$ has order 4 and $m=2$. If $j$ is even then $a^{i} b^{j}$ is a power of $a$ and $h_{\sharp}$ is not of order 3. This establishes that - for $m \neq 2, \mathcal{G}_{0}=Z_{2}$.

Finally suppose $m=2$. Then $\{\partial D\}=a^{2} b^{2 n}$ and $b^{2}$ has intersection number \pm 2 with $\{\partial D\}$ in $L$. Consequently there is a Möbius band $B$ embedded properly in $Y$ with $\partial B$ having the homotopy class $b^{2}$. But it is clear that another Möbius band $B_{1}$ can be chosen in $N$ with $\partial B_{1}=\partial B$. So $B \cup B_{1}$ gives a Klein bottle $K^{\prime}$ in $M$.

By Lemma 2, $M=N^{\prime} \cup Y^{\prime}$ where $N^{\prime}$ is a small regular neighbourhood of $K^{\prime}$ and $Y^{\prime}=M-$ int $N^{\prime}$ is a solid torus. Let $D^{\prime}$ be a meridian disk for $Y^{\prime}$. Then $\left\{\partial D^{\prime}\right\}=a_{0}^{m} b_{0}^{2 n}$ where $\pi_{1}\left(K^{\prime}\right)=\left\{a_{0}, b_{0} \mid b_{0}^{-1} a_{0} b_{0}=a_{0}^{-1}\right\}$, since the numbers $m, n$ are in 1-1 correspondence with the isomorphism class of the group $\pi_{1}(M)$. Therefore it is clear that a homeomorphism from $K$ to $K^{\prime}$ can be found which extends to $M$, and so $\mathcal{G}_{0}=S_{3}$.

For $m \neq 2, n=1$ we obtain $\mathcal{H}(M)=\mathcal{G}_{0}=Z_{2}$. If $m=2, n=1$ it follows that $\mathcal{H}(M)=\mathcal{G}_{0}=S_{3}$. Finally suppose $n \neq 1$. Then $\mathcal{H}(M)$ contains a sub- 
group $Z_{2}+Z_{2}$. Therefore if $m \neq 2, \mathcal{H}(M)=Z_{2}+Z_{2}$ and if $m=2$ then $\mathcal{H}(M)=S_{3}+Z_{2}$ since this is the only nonabelian group which has order 12 and contains a normal subgroup $Z_{2}$ (with quotient $S_{3}$ ).

Suppose $h: M \rightarrow M$ is an orientation-reversing homeomorphism. If $h_{\sharp} \in$ Aut $H_{1}\left(M, Z_{2}\right)$ is of order 3 then we replace $h$ by $h^{3}$. So it suffices to assume (by Theorem 4) that there is a Klein bottle $K$ in $M$, so that after an isotopy of $h, h(K)=K$. Then if we compose $h$ with a suitable orientation-preserving homeomorphism, a new $h$ is obtained with $h=$ id on $K$.

By the argument in the last paragraph of the proof of Theorem 4, we can adjust $h$ so that also $h: N \rightarrow N$. Then since $h$ is orientation-reversing, it must be the case that $h: L \rightarrow L$ is orientation-reversing. Suppose $h_{*}: \pi_{1}(L) \rightarrow \pi_{1}(L)$ maps $a$ to $a^{i} b^{j}$ and $b^{2}$ to $a^{q} b^{2 r}$. Since $h=$ id on $K$, it follows that in $\pi_{1}(K)$ the classes $a$ and $a^{i} b^{j}$ must be conjugate, and similarly for $b^{2}$ and $a^{q} b^{2 r}$. By a calculation in $\pi_{1}(K)$, one sees that $i= \pm 1, j=0, q=0$ and $r=1$. Then since $h: L \rightarrow L$ is orientation-reversing, we find that $i=-1$. But $h_{*}$ : $\pi_{1}(L) \rightarrow \pi_{1}(L)$ maps $\{\partial D\}$ to $\{\partial D\}^{ \pm 1}$, and $\{\partial D\}=a^{m} b^{2 n}$ for $m>0, n>0$. This gives a contradiction.

3. 2-groups acting freely on $S^{3}$. In [3] it is proved that a free action of $Z_{2}$ on $S^{3}$ is equivalent to an orthogonal action. We begin with a simple demonstration of:

Proposition 7 [5]. Any free action of $Z_{4}$ on $S^{3}$ is equivalent to an orthogonal action.

Proof. By [3], the quotient of $S^{3}$ by the action of the subgroup $Z_{2}$ of $Z_{4}$ is $R P^{3}$. Let $P$ be an embedded projective plane in $R P^{3}$. The action of $Z_{4}$ gives a free involution $g$ on $R P^{3}$.

Assume without loss of generality that $P$ and $g P$ are transverse (cf. the lemma in [5]). $P \cap g P$ contains a loop which is one-sided in $P$ and $g P$, and all the other components of $P \cap g P$ bound disks in both surfaces. This follows by Poincaré duality, since a one-sided curve in $P$ gives an element of $H_{1}\left(R P^{3}, Z_{2}\right)$ dual to the class in $H_{2}\left(R P^{3}, Z_{2}\right)$ corresponding to $g P$.

Suppose $C$ is a curve of $P \cap g P$ chosen so that $C$ bounds a disk $D$ in $g P$ with (int $D) \cap P=\varnothing$. Let $C=\partial D_{1}$ with $D_{1}$ in $P$. If $C$ is $g$-invariant then $D_{1}=g D$. Hence $D \cup D_{1}$ is a $g$-invariant sphere which bounds a $g$-invariant 3-cell in $R P^{3}$. By the Brouwer Fixed-Point Theorem, $g$ has a fixed-point in this cell, which is a contradiction. Therefore $C$ cannot be $g$-invariant and we can find a projective plane $P_{1}$ which is obtained by a small isotopy of $\left(P-\right.$ int $\left.D_{1}\right) \cup D$, so that $P_{1} \cap g P_{1}$ has fewer components than $P \cap g P$.

By this procedure we eventually reach a projective plane again denoted by $P$, with $P \cap g P$ a single curve. The complement of a small $g$-invariant regular neighbourhood of $P \cup g P$ in $R P^{3}$ consists of two 3-cells interchanged by $g$. 
So the action of $g$ is completely characterized and is equivalent to an orthogonal action.

TheORem 8. Suppose that $M$ is a 3-manifold as in Proposition 3. If there is a free involution acting on $M$ then the quotient is either a lens space or a manifold with the properties in Proposition 3.

Proof. Let $M=N \cup Y$ where $N$ is a small regular neighbourhood of a Klein bottle $K$ embedded in $M$. Let $g: M \rightarrow M$ be a free involution. We will show that the quotient has either an embedded Klein bottle or a genus 1 Heegaard splitting and this clearly implies the result.

Assume that $g K$ and $K$ are transverse. By exactly the same procedure as in Proposition 7, since $K$ and $g K$ are incompressible the contractible curves in their intersection can be eliminated. Suppose that a component $C$ of $K \cap g K$ is two-sided in $K$. If $T$ is a small regular neighborhood of $C$ in $M$ then $T-T \cap K$ has two components. Therefore $g K \cap(T-T \cap K)=(g K \cap$ $T)-C$ has two components, and this shows that $C$ is two-sided in $g K$.

Suppose next that $K \cap g K$ contains two or more two-sided (noncontractible) curves in $K$. If $C_{1}, C_{2}$ are loops of this type then clearly $C_{1} \cup C_{2}$ bounds annuli $A, A^{\prime}$ in $K, g K$ respectively. Without loss of generality assume $K \cap$ int $A^{\prime}=\varnothing$. Exactly one of the surfaces $(K-$ int $A) \cup A^{\prime}$ and $A \cup A^{\prime}$ is a Klein bottle, which we denote by $K_{1}$. Suppose $C_{1}$ is $g$-invariant and let $\pi$ : $M \rightarrow M_{0}$ be the quotient of $M$ by the action of $g$. By the argument on p. 14 of [13] (cf. also p. 44 of [12]) this case can only occur if $\pi\left(C_{1}\right)$ is orientation-reversing in $M_{0}$, i.e. $M_{0}$ is nonorientable. But $M_{0}$ is closed with finite fundamental group so this gives a contradiction.

Therefore neither $C_{1}$ nor $C_{2}$ can be $g$-invariant. If $C_{1} \neq g C_{2}$ then after separating $K_{1}$ slightly from $g K_{1}$, we see that $K_{1} \cap g K_{1}$ has less components than $K \cap g K$. On the other hand, if $C_{1}=g C_{2}$ then we can choose notation so that $g A=A^{\prime}$. In this case if $K_{1}=(K-$ int $A) \cup A^{\prime}$ then again after a small isotopy, $K_{1} \cap g K_{1}$ has fewer curves than $K \cap g K$. Finally, if $K_{1}=A \cup$ $A^{\prime}$ then $K_{1}$ is $g$-invariant and the result follows, since $M_{0}$ contains a Klein bottle.

So we have established that for suitable choice of $K, K \cap g K$ includes at most one two-sided curve. Assume $K \cap g K$ has exactly one such curve $C$. Then $C$ must be $g$-invariant, which gives a contradiction. Consequently it suffices to assume $K \cap g K$ contains only one-sided curves.

Case $1 . K \cap g K$ is a single curve $C$.

Let $T$ be a small $g$-invariant regular neighbourhood of $C$, so that $K \cap \partial T$ and $g K \cap \partial T$ are single curves, $C_{1}$ and $g C_{1}$ respectively. Let $A$ be an annulus on $\partial T$ between $C_{1}$ and $g C_{1}$. Then $K_{1}=(K-$ int $T) \cup A \cup(g K-$ int $T)$ is an embedded Klein bottle in $M$. Since $M_{0}$ is orientable, $g$ is orientation-pre- 
serving on $T$ and on $\partial T$. Therefore $A$ cannot be $g$-invariant, because $g$ interchanges the curves of $\partial A$. Consequently we can separate $K_{1}$ slightly from $g K_{1}$ so that $K_{1} \cap g K_{1}$ consists of two one-sided curves.

Case 2. $K \cap g K=C \cup g C$ (where $C$ is one-sided).

Let $T$ be a small regular neighbourhood of $C$ (with $T \cap g T=\varnothing$ ). Then $\pi(T)$ is a solid torus in $M_{0}$ with $\pi(K \cap T)$ equal to a properly embedded Möbius band. Let $K-$ int $T-$ int $g T=A$ and denote the closures of the components of $M-$ int $T-$ int $g T-K-g K$ by $Y_{1}$ and $Y_{2}$.

The well-known argument that a properly embedded, incompressible annulus in a solid torus is parallel into the boundary shows that either $Y_{1}$ or $Y_{2}$ is a solid torus, with a meridian disk $D_{1}$ which intersects $A$ and $g A$ each in a single arc. We choose notation so that this is true for $Y_{1}$. There are two possibilities:

(1) $Y_{1}$ and $Y_{2}$ are both g-invariant.

Let $C^{\prime}$ be a component of $\partial A$. Then $\pi\left(D_{1}\right)$ is a meridian disk for the solid torus $\pi\left(Y_{1}\right)$ (because $M_{0}$ is orientable) and the curves $\pi\left(C^{\prime}\right), \partial \pi\left(D_{1}\right)$ have intersection number \pm 2 in $\partial \pi\left(Y_{1}\right)$. So there is a Möbius band $B$ embedded properly in $\pi\left(Y_{1}\right)$ with $\partial B=\pi\left(C^{\prime}\right)$. Consequently $B \cup \pi(K \cap T)$ is a nonsingular Klein bottle in $\boldsymbol{M}_{\mathbf{0}}$.

(2) $g$ interchanges $Y_{1}$ and $Y_{2}$.

In this case both $Y_{1}$ and $Y_{2}$ are solid tori, with meridian disks $D_{1}$ and $g D_{1}$ which both cross $A$ and $g A$ each at single arcs. Therefore it is easy to see that $Y_{1} \cup Y_{2}$ is homeomorphic to $S^{1} \times S^{1} \times I$. Consequently by [9], $\pi\left(Y_{1} \cup Y_{2}\right)$ is homeomorphic to the twisted line-bundle over a Klein bottle. This proves that $M_{0}$ contains a Klein bottle.

Case 3. $K \cap g K=C_{1} \cup C_{2}$, with both curves $g$-invariant (and one-sided).

Let $T_{1}$ and $T_{2}$ be small $g$-invariant regular neighbourhoods of $C_{1}$ and $C_{2}$. Define $A, Y_{1}, Y_{2}$ as in Case 2, using $T_{1}$ and $T_{2}$ instead of $T$ and $g T$. Exactly as in Case 1, the two annuli on $\partial T_{1}$ between the curves $K \cap \partial T_{1}$ and $g K \cap \partial T_{1}$ cannot be $g$-invariant. Therefore it follows that $g: Y_{1} \rightarrow Y_{2}$ is the only possibility. As in (2) of Case 2 above, we find that $Y_{1} \cup Y_{2}$ is homeomorphic to $S^{1} \times S^{1} \times I$. Consequently the torus $\partial T_{1}$ gives a $g$-invariant Heegaard splitting of $M$. This establishes that $M_{0}$ has a Heegaard splitting of genus 1 and is a lens space.

COROllaRY 9. A free action of $Z_{8}$ or $Q\left(2^{k}\right), k>3$, on $S^{3}$ is equivalent to an orthogonal action.

Proof. Suppose first that $G=Z_{8}$ or $Q(8)$ and $G$ acts freely on $S^{3}$. Then there is a normal subgroup $Z_{4}$ of $G$ and by Proposition 7 , the quotient of $S^{3}$ by $Z_{4}$ is $L(4,1)$. Now this is a manifold of the type in Proposition 3 . Let $g$ be the free involution on $L(4,1)$ induced by the action of $G$ on $S^{3}$. Then by 
Theorem 8 , the quotient of $L(4,1)$ by $g$ is either a lens space or a manifold. with the properties in Proposition 3. But this is clearly the orbit space for the action of $G$ on $S^{3}$. Consequently the quotient of $S^{3}$ by $G$ is a Seifert manifold, by Proposition 3. Now the action of $G$ is equivalent to an orthogonal action if and only if its orbit space can be Seifert fibered (see [10]). Therefore the result is proved.

For $k>3$ the result follows by induction on $k$. Suppose $Q\left(2^{k}\right)$ acts freely on $S^{3}$. The action of the normal subgroup $Q\left(2^{k-1}\right)$ is equivalent to an orthogonal action by the inductive assumption. So the quotient of $S^{3}$ by the action of $Q\left(2^{k-1}\right)$, which we will denote by $M$, is a Seifert manifold. Now it is easy to show that because $\pi_{1}(M)=Q\left(2^{k-1}\right), M$ has $S^{2}$ as orbit surface and 3 exceptional fibres of multiplicity $2,2, p$, with $p>1$ (cf. [7] or[10]). Therefore $M$ is a manifold of the kind in Proposition 3. There is a free involution on $M$ induced by the action of $Q\left(2^{k}\right)$ on $S^{3}$. Then by Theorem 8 , the quotient of $M$ by the involution is a Seifert manifold. Since this is just the orbit space for $Q\left(2^{k}\right)$, the proof is complete.

\section{BIBLIOGRAPHY}

1. G. Bredon and J. Wood, Nonorientable surfaces in orientable 3-manifolds, Invent. Math. 7 (1969), 83-110. MR 39 \#7616.

2. D. Epstein, Curves on 2-manifolds and isotopies, Acta Math. 115 (1966), 83-107. MR 35 \#4938.

3. G. Livesay, Fixed point free involutions on the 3-sphere, Ann. of Math. 72 (1960), 603-611. MR 22 \#7131.

4. T. Price, Homeomorphisms of Quaternion space and projective planes in four space, J. Austral. Math. Soc. 23 (1977), 112-128.

5. P. Rice, Free actions of $Z_{4}$ on $S^{3}$, Duke Math. J. 36 (1969), 749-751. MR 40 \#2064.

6. G. Ritter, Free actions of $Z_{8}$ on $S^{3}$, Trans. Amer. Math. Soc. 181 (1973), 195-212. MR 47 \#9611.

7. H. Seifert, Topologie dreidimensionaler gefaserter Räume, Acta Math. 60 (1933), 147-238.

8. J. Stallings, On fibering certain 3-manifolds, Topology of 3-Manifolds and Related Topics, Prentice-Hall, Englewood Cliffs, N. J., 1962.

9. F. Waldhausen, On irreducible 3-manifolds which are sufficiently large, Ann. of Math. 87 (1968), 56-88. MR 36 \# 7146.

10. P. Orlik, Seifert manifolds, Lecture Notes in Math., vol. 291, Springer-Verlag, Berlin and New York, 1972.

11. J. H. Rubinstein, Free actions of some finite groups on $S^{3}$. I, Math. Ann. (to appear).

12. J. Hempel, 3-manifolds, Ann. of Math. Studies, no. 86, Princeton Univ. Press, Princeton, N. J., 1976.

13. J. Stallings, On the loop theorem, Ann. of Math. 72 (1960), 12-19. MR 22 \#2526.

Department of Mathematics, University of Mrlbourne, Parkville, Victoria 3052 , Australia 